\title{
Numerical Modeling of Two-Dimensional Gas-Liquid Flow Structures
}

\author{
Vladimir Evgenyevich Vershinin ${ }^{1}$, Rodion Mikhaylovich Ganopolsky ${ }^{2} \&$ Vitaly Olegovich Polyakov $^{3}$ \\ ${ }^{1}$ The Department of modeling of physical processes and systems, Tyumen State University, Tyumen, Russian \\ Federation \\ ${ }^{2} \mathrm{PhD}$., head of Main computer center, Tyumen State University, Tyumen, Russian Federation \\ ${ }^{3}$ Science and technology park, Tyumen State University, Tyumen, Russian Federation \\ Correspondence: Vladimir Evgenyevich Vershinin, The Department of modeling of physical processes and \\ systems, Tyumen State University, 625003, Semakova str.10, Tyumen, Russian Federation. Tel: \\ 7-891-2928-7236. E-mail: vvershinin@list.ru. Rodion Mikhaylovich Ganopolsky, Main computer center, \\ Tyumen State University, 625003, Tyumen, Russian Federation. E-mail: rodion@utmn.ru
}

\author{
Received: November 10, $2014 \quad$ Accepted: November 13, $2014 \quad$ Online Published: January 15, 2015 \\ doi:10.5539/mas.v9n2p236 \\ URL: http://dx.doi.org/10.5539/mas.v9n2p236
}

The research is financed by the Ministry of Education and Science of the Russian Federation within the project by Government Order No 218 dd April 9 $9^{\text {th }}$, 2010, by contract No 02. G 25.31.0020 JSC "HMS Neftemash" in collaboration with FSBEI HPE Tyumen State University.

\begin{abstract}
In this paper the results of the numerical modeling of two-phase gas and liquid flows are given in horizontal channels for the purpose of definition of occurring flow structures and their existence conditions. The calculations were performed for two-dimensional models. The regimes occurring at the velocity range from 0.1 $\mathrm{m} / \mathrm{s}$ to $15 \mathrm{~m} / \mathrm{s}$ and various phase relation were under investigation. The phase boundaries location in the flow was determined with the VOF- volume of fluid method. The discovered flow structures were qualitatively analyzed and identified due to the accepted classification. Parameters of the modeling regimes were compared to the semi-empirical regime map of Taitel and Dukler, whereby the expected flow type was determined. The area of stream structures coincidence was defined in the numerical modeling using the regime map of Taitel and Dukler.
\end{abstract}

Keywords: multiphase current, numerical modeling, card of modes of a current, OpenFoam, volume of fluid method

\section{Introduction}

When creating various devices processing multiphase mixtures the issue of the investigated stream structure comes up. The structure of multiphase stream flow depends on the ratio volumes of phases, overall volumetric flax, channel orientation and geometry, flow direction (downflow, upflow, horizontal flow) as well as on the fluid and gas properties inside the channel. However the issue of the mentioned properties influence on the steady flow type is open. In the overwhelming majority of cases the multiphase streams are observed in round pipes and the question of stream section shape influence on the steady flow type is open. To reduce the impact of channel geometry we offer to consider a two-dimensional flow limit case. Among the others the flow dimensionality factor is of interest. On the one hand, two-dimensional flows are applied practically and investigation of two-dimensional structures properties versus three-dimensional is a separate task. On the other hand, detection of conditions under which the qualitative uniformity of two- and three-dimensional flow structures is observed would provide an opportunity to apply techniques of accelerated estimations of flow parameters practically for engineering numerical calculations. The issue of estimation time reduction is of high relevance for design of some technical devices. The issue of the mathematical model adequacy is very important as well.

\section{Review of Literature}

\subsection{Types of Two-Phase Flows}

By an experimental approach we have established a huge amount of gas-liquid flows structures in channels different in phase interface shape (Beggs H. D., Brill J. P., 1973). The regime classification based on its visual perception is reasonably subjective. The basic structures for horizontal three-dimensional channels are bubble, 
plug, emulsive, annular-dispersed and stratified structures (fig. 1). Thereat plug and emulsive structures are sometimes combined into intermitted regime. The stratified structure is divided into a properly stratified regime with no waves in phase interface and a wave regime (1). Due to experimental researches the additional fluidal regimes were also discovered in 2D channels. (Chinnov E.A., Kabanov O.A., 2008, Serizava A., Feng Z.,Kawara Z., 2002, Xu J.L. et al.1999, Abiyev R.S., Lavretsov I.V., 2010, Lavretsov I.V., 2009 ). It is clear to see from the qualitative description of two-phase stream specific structures the importance of correct definition of these structures when calculating the flow resistance and heat exchange. To define the expected flow structure (regime) the maps integrating data graphically and visualizing domains of structures existence in terms of stream conditions are extensively used. (Mandhane, J. M. et al. 1974, Schicht H.H., 1969, Scott D. S., 1963, Taitel Y., Dukler A. E., 1976). The maps are different in selecting variables that define the regime.

a)

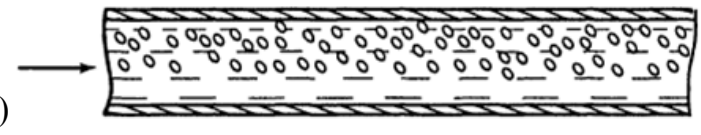

b)

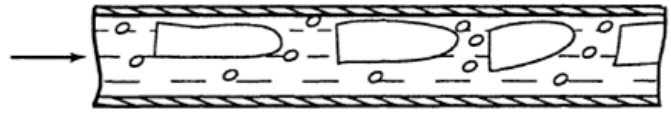

c)

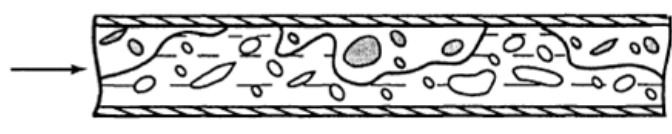

d)

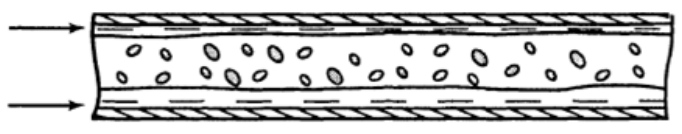

e)

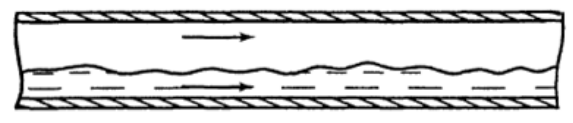

Figure 1. Structures of gas-liquid flow in horizontal pipes [1]: a - bubble; b - plug; $\mathrm{c}$ - emulsive; $\mathrm{d}-$ annular-dispersed; e- stratified

However it should be kept in mind that the flow regime boundaries depend both on the operating conditions and phase properties, and on channel inlet conditions. Thereat, the evaluation of a two-phase flow structure takes place at the long length, exceeding 100 channel diameters in many cases. Transfer from one structure to the other is caused by the buckling failure of the original structure. Therefore the two-phase stream regime maps should be considered as the tools for the approximate estimation of the flow type.

So let us consider the regime map of Taitel and Dukler (Taitel Y., Dukler A. E., 1976.) Generalized stream parameters are used to determine the flow regime, so that the map can be applied for wide liquids range and various pipe sizes. The flow regime map is shown in the figure 2. A boundary between the wavy regime and the others is determined with the Froude number $F$ and the Lockhart-Martinelli parameter $X$ (Beggs H. D., Brill J. P., 1973.):

$$
\begin{aligned}
& F=w_{g} \sqrt{\frac{\rho_{g}}{\left(\rho_{l}-\rho_{g}\right) g D}} \\
& X=\sqrt{\left(\frac{d p}{d z}\right)_{\text {liquid }} /\left(\frac{d p}{d z}\right)_{\text {gas }}}
\end{aligned}
$$

where $F$ is the Froude number; $X$ is the Lockhart-Martinelli parameter; $\rho_{g}$ and $\rho_{l}$ are gas and liquid densities; $g$ is the gravity factor module; $D$ is the channel diameter; $w_{g}$ is the superficial gas velocity 
(calculated by gas rate); $\left(\frac{d p}{d z}\right)$ liquid and $\left(\frac{d p}{d z}\right)$ gas are the pressure gradients in the channel where either the liquid phase or gas phase flows with its actual discharge.

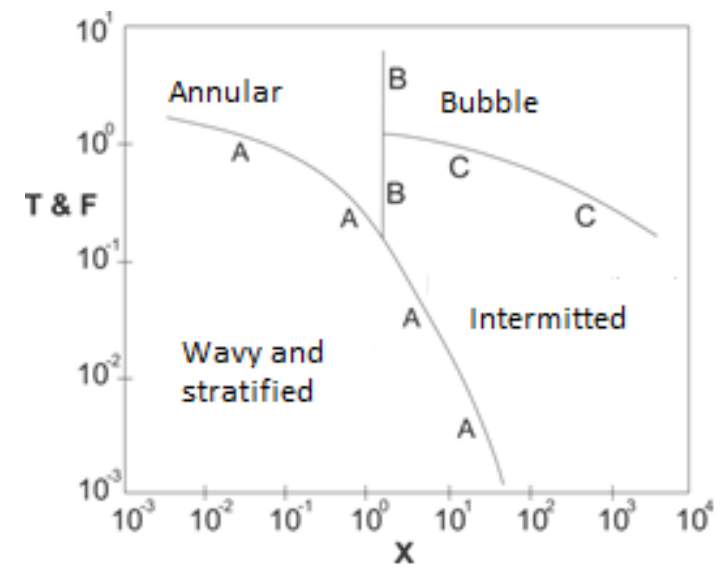

Figure 2. The flow regime map of Taitel and Dukler for horizontal channels (Taitel Y., Dukler A. E., 1976). A and $\mathrm{B}$ boundaries are determined with the variables $\mathrm{X}$ and $\mathrm{F}, \mathrm{C}$ boundary is determined with the variables $\mathrm{X}$ and $\mathrm{T}$

For the boundary of the bubble or intermitted regime upon annular the X parameter is constant and equals 1.6. The boundary of the bubble regime upon intermitted is determined by the $\mathrm{X}$ parameter and the $\mathrm{T}$ number that could be considered as the Froude number alternative and determined from the liquid phase velocity:

$$
T=\sqrt{\frac{\left(\frac{d p}{d z}\right)_{\text {liquid }}}{\left(\rho_{l}-\rho_{g}\right) g}}
$$

According to the approximate calculation procedure for conditions of relative transition among the various stream structures the stratified flow regime is considered as the basic regime for the flow in horizontal and low inclined pipes. For this structure the one-dimensional momentum conversation equations are written separately for the liquid and gas streams. When the friction law is prescribed such approach enables to calculate the sectional fractions at the interphase boundary for every phase within the observed flow regime as well as the pressure gradient in the pipe. Supposing the liquid and gas were flowing in a pipe with their mass rate in absence of the other phase, the respective pressure gradients would be expressed by the famous Darcy-Weisbach's law at the cost of friction (Loytsyanskiy L. G., 2003).

$$
-\left(\frac{d p}{d z}\right)=\frac{\lambda}{2} \frac{\rho w}{D}
$$

where $\lambda$ is the hydraulic drag coefficient, $D$ is the hydraulic diameter, $\rho$ is the phase density, $w$ is the phase velocity. The Lockhart-Martinelli parameter (2) based on the ratio of phase pressure gradients in a pipe shows that the two-phase mixture behaves much similar to the liquid, than to the gas.

The drag coefficients are calculated as the Reynold's numbers functions $R e_{l}$ and $R e_{g}$ using well-known formulas from mechanics of single-phase systems. For liquid and gas these numbers are determined with the following formulas (Loytsyanskiy L.G., 2003):

$$
R e_{l}=\frac{w_{l} \rho_{l} D}{\mu_{l}} \quad R e_{g}=\frac{w_{g} \rho_{g} D}{\mu_{g}}
$$

where $\mu_{l}, \mu_{g}$ are the dynamic viscosities of gas and liquid properly.

\subsection{Mathematical Model of Two-Phase Flow}

Let us consider two-phase two-dimensional isothermal flow of the viscous liquid and gas. To define the multiphase flow structure we should determine the location of the phase boundaries in a stream. We take the phases as incompressible. This condition is correct when the pressure gradients in the stream are insignificant. The motion of every phase in such a case obeys the Navier-Stokes equation: 


$$
\frac{d}{d t}(\rho \vec{V})=-\nabla p+\rho \vec{g}+\vec{F}+\nabla(\mu \nabla \vec{V})
$$

where $\rho$ is the phase density, $p$ is the pressure, $\mu$ is the dynamic phase viscosity, $\vec{V}$ is the phase motion velocity, $\vec{g}$ is the gravity factor, $\vec{F}$ is the body force vector. The interphase boundary occurrence imposes some additional conditions such as absence of boundary overflow and momentum conversation at the boundary. The estimation of free interphase boundary location is a nontrivial task the can be solved with a number of numerical procedures. (Ferziger J. H., Peric M. ,2002, Harlow F. H., Welch J. E. ,1965, Scardovelli R., Zaleski S., 1999, Osher S.J., Fedkiw R.P., 2003, Strakhovskaya L.G., 2009, Strakhovskaya L.G., 2010, Rukavishnikov A.B., 2008). We consider the VOF-method based on single-fluid approximation as one of the approached widely applied to track out the boundary between gas and liquid. (Hirt C. W., Nicolls B. D.,1981). Herewith the scalar function $\alpha$-phase volume content - is to observe.

$$
\alpha=\left\{\begin{array}{c}
1, \text { the phase is equal to the cell volume } \\
0, \text { the phase is not equal to the cell volume } \\
(0 ; 1) \text {, there is a phase interface in the cell }
\end{array}\right.
$$

The $\alpha$ development is determined by the transfer equation, represented by the conservation law of the phase volume ratio:

$$
\frac{\partial \alpha}{\partial t}+\vec{V} \cdot \nabla \alpha=S_{s}
$$

where $S_{s}$ specifies the phase source (run-off) strength. Within this approach the condition of zero crossflow is always fulfilled at the phase boundary. Flow of liquid and gas, interacting through the interphase boundary, is substituted for mono-phase flow, described by the density and pressure jump at the boundary. In such a case there is one equation (6) for the whole stream to solve. The viscosity and density are determined as the formula

$$
\mu=\alpha \mu_{1}+(1-\alpha) \mu_{2} ; \rho=\alpha \rho_{1}+(1-\alpha) \rho_{2}
$$

where $\rho_{1}, \rho_{2}$ are the densities of the $1^{\text {st }}$ and $2^{\text {nd }}$ phases, $\mu_{1}, \mu_{2}$ are the dynamic viscosities of the $1^{\text {st }}$ and $2^{\text {nd }}$ phases, $\alpha$ is the volume content of the $1^{\text {st }}$ phase. As one of the VOF-method advantages we can select the analysis grid constancy and relative calculation simplicity as well.

\section{Numerical Modeling Methods for Two-Phase Flow}

The hydrodynamical simulator OpenFOAM with a plain code was used for modeling of two-phase gas-liquid flows (Kurbanaliyev A. Y., Tayirov M. M., 2011, www.thermalfluidscentral.org, www.thermopedia.com). We performed all the calculations using the supercomputer. To complete the task we used based on the VOF-method Multiphase InterFOAM for incompressible, isothermal, immiscible liquids.

The system of original differential equations can be transformed into system of the linear algebraic equations with the help of the existing numerical circuits (Fletcher C., 1991). The Gaussian linear spatial discretization circuit was applied in this paper. The obtained algebraic equation system was solved with the conjugate gradient method (Ferziger J. H., \& Peric M., 2002).

All the fluids densities and viscosities were taken as constant and irrespective of the pressure when calculating. The gas was taken as the first phase so that $\alpha$ presented the volumetric gas content.

As it was said, a stable stream structure is formed gradually, for this reason to obtain the correct results the calculations should be done for extended sections. Therefore the quantity of calculated cells in a model can reach $10^{6}$, and the calculation time comes up to 10-200 hours using the supercomputer. The calculations were made for the two-dimensional model of channel, limited with the upper and lower boundaries. The following stream properties were used when modeling:

The channel height is $0.04 \mathrm{~m}$, the hydraulic diameter of the channel corresponds to the pipe diameter $0.08 \mathrm{~m}$ upon that. The stream velocity range is from $0.1 \mathrm{~m} / \mathrm{s}$ to $15 \mathrm{~m} / \mathrm{s}$. The channel length was chosen according to the range velocity within the limits $1-100 \mathrm{~m}$ to ensure at least 10 second stream flow time in the channel. The cell size in the analysis grid is $1,8 \mathrm{~mm}$. The cells are square-shaped. The gas content range is from 0.1 to 0.9 in increments of 0.2 . Physical properties of the phases correspond to oil and air: the liquid density is $820 \mathrm{~kg} / \mathrm{m}^{3}$, the gas density is $12 \mathrm{~kg} / \mathrm{m}^{3}$, the kinematic liquid viscosity is $4 \cdot 10^{-5} \mathrm{~m}^{2} / \mathrm{s}$, the kinematic gas viscosity is 1,48 . $10^{-5} \mathrm{~m}^{2} / \mathrm{s}$. At the channel inlet $\alpha$ distribution corresponds to the max homogeneous stream structure. The $\alpha$ value in cells takes on 2 possible values: 1 or 0 . The $\alpha$ values distribute in cells in a random manner. $\alpha$ distribution consistency allows to avoid the influence of initial conditions on the modeling results. Flow homogeneity at the channel inlet prevents possible correlations when the flow develops from one structure to another. The stream 
velocity and zero pressure gradient were assigned at the channel inlet. At the outlet the condition of free channel discharge and the constant pressure were assigned for the velocity.

\section{Results}

According to the calculation results flow longitudinal sections for all 35 estimations were constructed, and with that the qualitative evaluation of stream regime was made. The patterns of the basic observed flow regimes and type evaluation are given in the figures 3-6. The gas is marked with blue and the liquid with red. To minimize the influence of the initial and boundary conditions the stream regime was estimated at a distance equal to 10 second of mixture lifetime. The channels given in the figures are cut into sections and located one below the other for reasons of compactness. Fragments of the fig. 1 with the appropriate regime are also shown in the figures below.

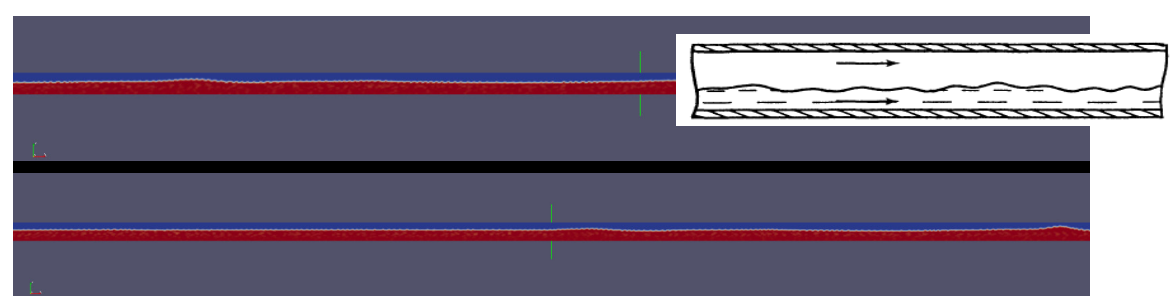

Figure 3. The stratified flow regime (stream velocity $u=0.5 \mathrm{~m} / \mathrm{s}, \alpha=0.5$ )

a)

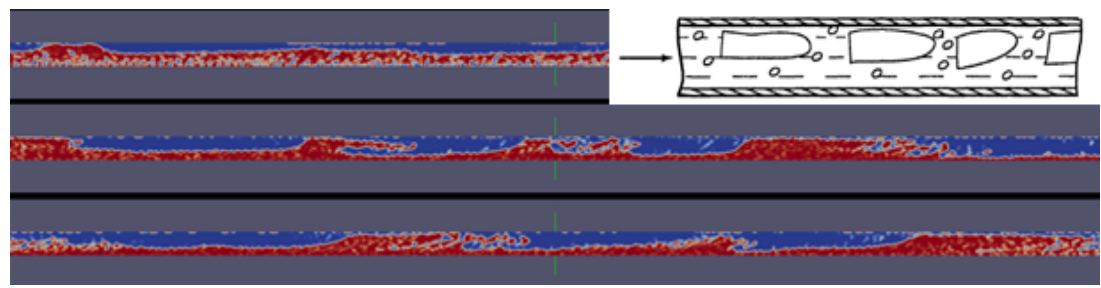

Figure 4. The intermitted flow regime. $\mathrm{a}$ - the flow regime is close to the stratified or plug regime (stream velocity $u=2 \mathrm{~m} / \mathrm{s}, \alpha=0.3$ ) , $b$ - the flow regime is close to the emulsive (stream velocity $u=5 \mathrm{~m} / \mathrm{s}, \alpha=0.3$ )

b)
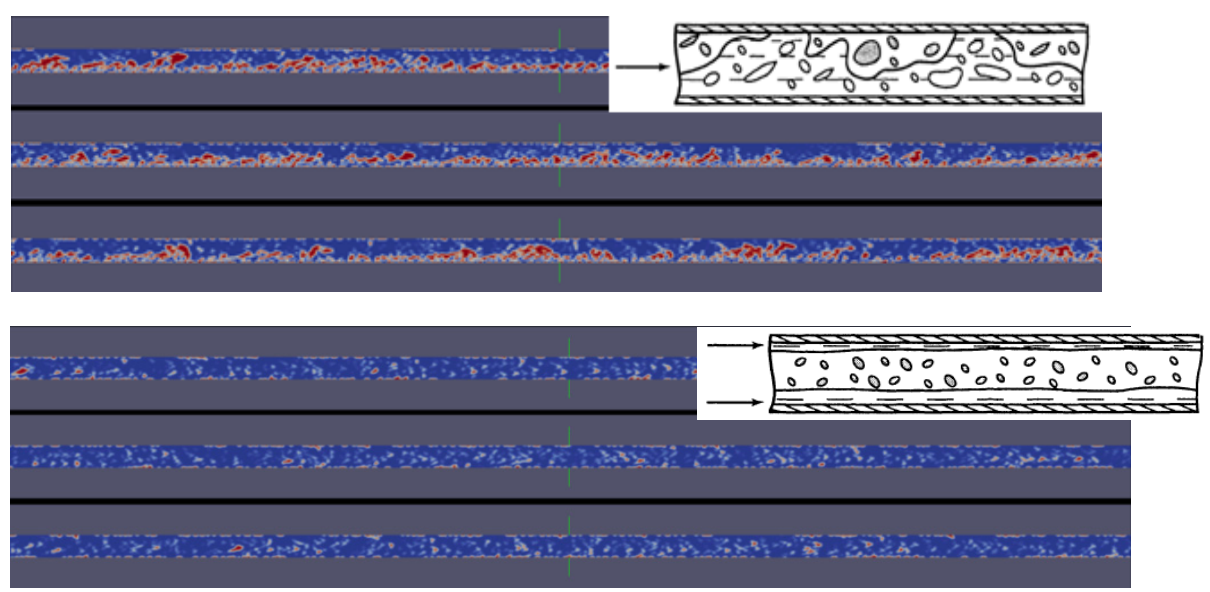

Figure 5. The annular flow regime (stream velocity $\mathrm{u}=10 \mathrm{~m} / \mathrm{s}, \alpha=0.9$ )

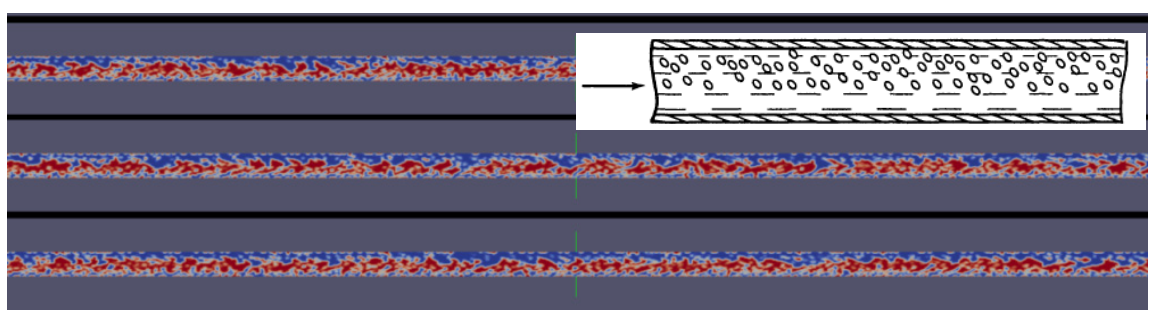

Figure 6 . The bubble flow regime. (stream velocity $\mathrm{u}=15 \mathrm{~m} / \mathrm{s}, \alpha=0.3$ ) 


\section{Discussion}

As it appears from the figures 3-6, all the structures specific to 3-dimensional 2-phase flows are actualized in 2-dimensional 2-phase gas-liquid flows. This goes to prove that the force nature and order in 2- and 3-dimensional flows are fast similar when modeling. Therefore the bubble regime requires a dense grid to make the small bubbles visible. The phase distribution pattern presented in the figure 6 is close to the bubble regime, but differs in cell sizes $(1.8 \mathrm{~mm}$ ). In case the channel is long (as a result of high stream velocity) crosspartition of the cells leads to increase of rated cells number and calculation time.

The flow regime map constructed for three-dimensional horizontal two-phase flows can be used to evaluate the coincidence rate of every regime existence conditions for two- and three- dimensional flows.

For purpose of comparison of the obtained regimes estimations to the Taitel and Dukler's map we constructed the tables below. The Table 1 represents the F, X, T parameters determined by the formulas (1)-(3) and the Table 2 shows the comparison of the flow regimes predicted with the map and the actually evaluated. The lines in the Table 2 marked with 'OF' represent the flow pattern estimation, resulted from the OpenFoam numerical modeling. The lines marked with 'T\&D' represent the estimation based on the Taitel and Dukler's map. The empty cells reveal the complexity of single classification of the obtained stream regime. The values denoted with an asterisk evidence that the stream regime is close to the transitional boundary. As it appears from the Table 2 the predicting three-dimensional flow regime is in accord with the modeling two-dimensional flow regime when the stream velocities are within the range $2-5 \mathrm{~m} / \mathrm{s}$. The highest regime difference is observed at high gas content $(\alpha=0.9)$ within the whole velocities range (Table 2, right column) as well as at some low and high stream velocities $(\mathrm{u}=0.1-1.5 \mathrm{~m} / \mathrm{s}$ and $\mathrm{u}=10-15 \mathrm{~m} / \mathrm{s}$ ) (Table 2, opening and finishing lines).

Table 1. Parameters for the modeling flows at different regimes: $\mathrm{X}$ is the Lockhart-Martinelli parameter, $\mathrm{F}$ is the Froude number, $\mathrm{T}$ is the Taitel and Dukler's parameter

\begin{tabular}{rrrrrrr}
\hline & & \multicolumn{3}{l}{$\alpha$} \\
$\mathrm{U}, \mathrm{m} / \mathrm{s}$ & & 0,1 & 0,3 & 0,5 & 0,7 & 0,9 \\
\hline & $\mathbf{X}$ & 40,7696 & 20,7589 & 13,5899 & 8,8967 & 4,5300 \\
0,1 & $\mathbf{F}$ & 0,0014 & 0,0041 & 0,0069 & 0,0096 & 0,0124 \\
& $\mathbf{T}$ & 0,0432 & 0,0381 & 0,0322 & 0,0249 & 0,0144 \\
& $\mathbf{X}$ & 40,7696 & 20,7589 & 13,5899 & 8,8967 & 4,2363 \\
0,5 & $\mathbf{F}$ & 0,0069 & 0,0206 & 0,0344 & 0,0481 & 0,0619 \\
& $\mathbf{T}$ & 0,0965 & 0,0851 & 0,0719 & 0,0557 & 0,0322 \\
& $\mathbf{X}$ & 40,7696 & 20,7589 & 11,2613 & 5,8524 & 2,6748 \\
1 & $\mathbf{F}$ & 0,0138 & 0,0413 & 0,0688 & 0,0963 & 0,1238 \\
& $\mathbf{T}$ & 0,1365 & 0,1203 & 0,1017 & 0,0788 & 0,0455 \\
& $\mathbf{X}$ & 60,4345 & 18,5640 & 7,7034 & 4,4422 & 2,0584 \\
2 & $\mathbf{F}$ & 0,0275 & 0,0825 & 0,1376 & 0,1926 & 0,2476 \\
& $\mathbf{T}$ & 0,2861 & 0,2124 & 0,1438 & 0,1114 & 0,0643 \\
& $\mathbf{X}$ & 72,2072 & 19,6453 & 9,3458 & 4,1632 & 1,4598 \\
5 & $\mathbf{F}$ & 0,0688 & 0,2063 & 0,3439 & 0,4815 & 0,6190 \\
& $\mathbf{T}$ & 0,6522 & 0,5234 & 0,3893 & 0,2328 & 0,1017 \\
& $\mathbf{X}$ & 64,0563 & 19,6458 & 9,3603 & 4,4588 & 1,1257 \\
10 & $\mathbf{F}$ & 0,1376 & 0,4127 & 0,6878 & 0,9629 & 1,2381 \\
& $\mathbf{T}$ & 1,1961 & 0,9600 & 0,7151 & 0,4573 & 0,1438 \\
& $\mathbf{X}$ & 64,0110 & 19,6458 & 9,3603 & 4,4598 & 1,2777 \\
15 & $\mathbf{F}$ & 0,2063 & 0,6190 & 1,0317 & 1,4444 & 1,8571 \\
& $\mathbf{T}$ & 1,7054 & 1,3688 & 1,0197 & 0,6522 & 0,2328 \\
\hline
\end{tabular}

Table 2. The interface of the Taitel and Dukler's flow regime map and the results of 2-dimensional flows modeling

\begin{tabular}{rrrrrrr}
\hline & & \multicolumn{9}{c}{$\alpha$} \\
U, m/s & & 0,1 & 0,3 & 0,5 & 0,7 & 0,9 \\
\hline \multirow{2}{*}{0,1} & T\&D: & SW $^{*}$ & SW $^{*}$ & SW $^{*}$ & SW $^{*}$ & SW $^{*}$ \\
& OF: & SW & SW & SW & SW & SW \\
0,5 & T\&D: & I & I & I & I & SW $^{*}$ \\
& OF: & - & SW & SW & SW & SW \\
& T\&D: & I & I & I & I & I $^{*}$ \\
& OF: & SW & SW & SW & SW & SW \\
& T\&D: & I & I & I & I & $I^{*}$ \\
& OF: & SW & SW & SW & SW & SW \\
\hline
\end{tabular}




\begin{tabular}{ccccccc}
\hline \multirow{2}{*}{5} & T\&D: & I & I & I & I & I $^{*}$ \\
10 & OF: & I & I & I & SW & SW \\
& T\&D: & I & I & I & I & AD $^{*}$ \\
& OF: & I & I & I & I & SW \\
15 & T\&D: & DB & DB $^{*}$ & I & I & AD $^{*}$ \\
& OF: & & DB & & & AD \\
\hline
\end{tabular}

Reference designations for the flow regimes: AD-annular-dispersed; DB- dispersed bubble; I-intermitted; SW-stratified wavy.

It should be taken into account that the flow parameters at the right column of the Table 2 corresponds to transition section at the Taitel and Dukler's map near the lines A and B as it is shown in the figure 8, constructed according to the Table 1 data.

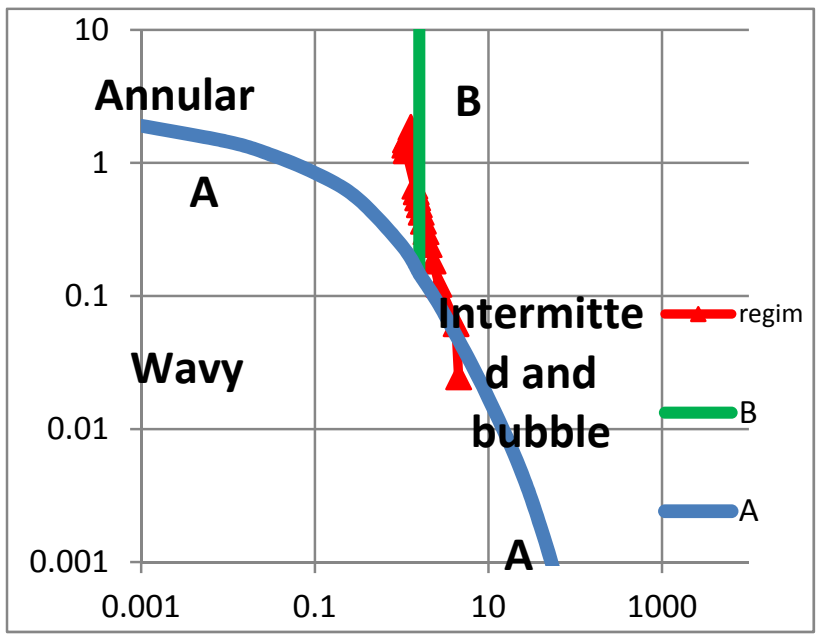

Figure 8. Correspondence of the stream flow regimes, when $\alpha=0.9$ within the velocity range $\mathrm{u}=0,1-15 \mathrm{~m} / \mathrm{s}$ according to the Taitel and Dukler's flow regime map for horizontal channels

The numerical modeling and flow type explanation are complicated in this section. The differences in the other cases can be also caused by appropriation of the flow conditions to the transition areas (in the range of $\mathrm{A}$ and $\mathrm{C}$ boundaries, fig.2). Far from the transition areas we can observe the coincidence of the flow regimes of the modeling two-dimensional and real three-dimensional flows. Overall we can conclude that two-dimensional and three-dimensional flows are identical in respect of the qualitative characteristics and existence conditions of the various structures. This allows us to apply two-dimensional calculations to evaluate some properties of two-phase flows in three-dimensional cases.

\section{Conclusion}

Although the modeling results showed that the numerical calculations of two-dimensional two-phase horizontal flows inside the channels based on the VOF-method represent almost all observed by the experiment flow types, it is reasonable to notice that the area under investigation appeared to be not wide enough. We explain it with the computational complexity of the modeling high-velocity flows. The estimations of flows in vertical channels are of particular interest. If we research all types of the channel orientation, we could make clear the construction mechanism of a particular structure, as well as analyze the influence of stream dimensionality on the flow regime.

The authors are grateful to the staff of the Main Computer Center and the Technology Park of the Tyumen State University, who lent their countenance and technical support to this research.

\section{Acknowledgement}

The work was supported by the Ministry of Education and Science of the Russian Federation within the project by Government Order No 218 dd April $9^{\text {th }}$, 2010, by contract No 02. G 25.31.0020 JSC "HMS Neftemash" in collaboration with FSBEI HPE Tyumen State University.

\section{References}

Abiyev, R. S., \& Lavretsov, I. V. (2010). Hydrodynamics of Taylor's gas-liquid mixture flow in microchannels: 
Theory and experiment. Abstracts of XIII internat. conf. "Hi-tech chemical technologies - 2010". - Ivanovo: IGHTU, p. 45.

Beggs, H. D., \& Brill, J. P. (1973). A Study of Two-Phase Flow in Inclined Pipes. JPT (May 1973) 607; Trans, AIME, 255.

Chinnov, E. A., \& Kabanov, O. A. (2008). Two-phase flow regimes in flat short microchannel. Letters to GTF, 34(16), 41-46.

Ferziger, J. H., \& Peric, M. (2002). Computational Methods for Fluid Dynamics. Berlin: Springer Verlag, 423. http://dx.doi.org/10.1007/978-3-642-56026-2

Fletcher, C. (1991). Computational Techniques for Fluid Dynamics: In, 2, transl. from Engl. - Moscow: Mir, 552.

Harlow, F. H., \& Welch, J. E. (1965). Numerical Calculation of Time-Dependent Viscous Incompressible Flow of Fluid with Free Surface. The Physics of Fluids, 8, 2182-2189. http://dx.doi.org/10.1063/1.1761178

Hirt, C. W., \& Nicolls, B. D. (1981).Volume of fluid (VOF) method for dynamics of free boundaries. Journal of Computational Physics. 39, 201-225. http://www.thermopedia.com/content/2, https://www.thermalfluidscentral.org/encyclopedia/index.php/Two-Phase_Flow_Patterns_in_Horizontal_Tu bes

Kurbanaliyev, A. Y., \& Tayirov, M. M. (2011). OpenFOAM for modeling of flows with free boundaries. News of KGTU n.a. I. Razzakov. No24. - Bishkek, p. 421-424.

Lavretsov, I. V., Aldoshin, A. S., \& Abiyev, R. S. (2009). Regimes of gas-liquid mixture flow in monolithic catalyst channels. Collection of scientific papers "Kataliz: yesterday, today, tomorrow". - S. Pb: SPBGTI (TU), P.133-136. http://dx.doi.org/10.1016/0021-9991(81)90145-5

Loytsyanskiy, L. G. (2003). Liquid and Gas Mechanics. Moscow: Drofa, 840p.

Mandhane, J. M., Gregory, G. A., \& Aziz, K. (1974). A Flow Pattern Map for Gas-Liquid Flow in Horizontal Pipes. Intern. J. Multiphase Flow, 1, 537-553. http://dx.doi.org/10.1016/0301-9322(74)90006-8

Osher, S. J., \& Fedkiw, R. P. (2003). Level Set Method and Dynamic Implicit Surfaces.N.Y. Springer, 273. http://dx.doi.org/10.1007/b98879

Rukavishnikov, A. B. (2008). Generalized statement of the problem of two-phase liquid flow with continually varying interface. Mathematical modeling, 20(3), 3-8.

Scardovelli, R., \& Zaleski, S. (1999). Direct Numerical Simulation of Free-Surface and Interfacial Flow. Annual Review of Fluid Mechanics, 31, 567. http://dx.doi.org/10.1146/annurev.fluid.31.1.567

Schicht, H. H. (1969). Flow patterns for an adiabatic two-phase flow of water and air within a horizontal tube. Verfahrenstechnik, 3(4), 153-161.

Scott, D. S. (1963). Properties of concurrent gas-liquid flow. Adv. Chem. Engng., 4, 199-277. http://dx.doi.org/10.1016/S0065-2377(08)60241-0

Serizava, A., Feng, Z., \& Kawara, Z. (2002). Thermal Fluids Science, 26, 703-714. http://dx.doi.org/10.1016/S0894-1777(02)00175-9

Strakhovskaya, L. G. (2009). About one of MKSE variants for estimation of viscous incondensable liquid flows. G. calcul. math. and math. phys., 49(1), 123-136.

Strakhovskaya, L. G. (2010). Numerical modeling of two-phase flows with moving interface based on the resulting super-elements method. Preprints of IMP n.a. M.V. Keldysh., (83), 12.

Taitel, Y., \& Dukler, A. E. (1976). A Model for Predicting Flow Regime Transition in Horizontal and Near Horizontal Gas-Liquid Flow. AlChE J., 22(47-55). http://dx.doi.org/10.1002/aic.690220105

Xu, J. L., Cheng, P., \& Zhao, T. S. (1999). Multiphase Flow, 25, 411-432. http://dx.doi.org/10.1016/S0301-9322(98)00057-3

\section{Copyrights}

Copyright for this article is retained by the author(s), with first publication rights granted to the journal.

This is an open-access article distributed under the terms and conditions of the Creative Commons Attribution license (http://creativecommons.org/licenses/by/3.0/). 